Case Report

\title{
Donor Cell-Derived Acute Myeloid Leukaemia with 3q26.2 Involvement/MECOM Rearrangement - A Case Report and Literature Review
}

Jun Gu ${ }^{1}$, Wei Wang ${ }^{2}$, Guilin Tang ${ }^{2}$, Gokce A. Toruner ${ }^{2}$, Ming Zhao ${ }^{1}$, Steven Sfamenos ${ }^{1}$, Zhenya Tang $^{2, *}$, Joseph D. Khoury ${ }^{2}$, L. Jeffrey Medeiros ${ }^{2}$

1. School of Health Professions, the University of Texas MD Anderson Cancer Centre, 1515 Holcombe Blvd., Houston, USA; E-Mails: jungu@mdanderson.org; mzhao@mdanderson.org; SMSfamenos@mdanderson.org.

2. Department of Hematopathology, the University of Texas MD Anderson Cancer Centre, 1515 Holcombe Blvd., Houston, USA; E-Mails:WWang13@mdanderson.org; GTang@mdanderson.org; GAToruner@mdanderson.org; ztang@mdanderson.org; JKhoury@mdanderson.org; ljmedeiros@mdanderson.org.

* Correspondence: Zhenya Tang; E-Mail: ztang@mdanderson.org

Academic Editor: Gerhard Hildebrandt

Special Issue: $\underline{\text { Bone Marrow Transplantation }}$

OBM Transplantation

2020 , volume 4 , issue 4

doi:10.21926/obm.transplant.2004128
Received: October 13, 2020

Accepted: November 29, 2020

Published: December 7, 2020

\begin{abstract}
Donor cell-derived leukaemia (DCL) is an uncommon complication of allogeneic hematopoietic stem cell transplantation (HSCT). DCL might represent up to $5 \%$ of the postHSCT disease relapses, but case numbers reported in the literature might underestimate the frequency. The leukemogenesis of $D C L$ is not well understood due to the limited numbers of cases reported and lack of detailed molecular genetic information from recipients and donors. Although many theories have been proposed for leukemogenesis of $\mathrm{DCL}$, the underlying molecular genetic mechanism are likely heterogeneous. Here we report a case of donor cellderived acute myeloid leukaemia with 3q26.2 involvement/MECOM rearrangement and
\end{abstract}

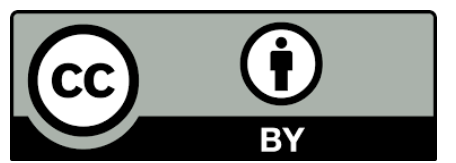

(C) 2020 by the author. This is an open access article distributed under the conditions of the Creative Commons by Attribution License, which permits unrestricted use, distribution, and reproduction in any medium or format, provided the original work is correctly cited. 
chromosome 20 q deletion. We also reviewed the literature of previously described DCL cases, and we discussed the risk factors that might be important to the onset of DCL.

\section{Keywords}

Donor cell leukaemia; hematopoietic stem cell transplantation (HSCT); 3q26.2 involvement/MECOM rearrangement; del20q; leukemogenesis

\section{Introduction}

Donor cell-derived leukaemia $(D C L)$ is a rare complication of allogenic hematopoietic stem cell transplantation (HSCT). DCL might represent up to $5 \%$ of the post-transplant relapsed leukaemia cases [1-4]. To date, less than 200 DCL cases have been reported since its first recognition in 1971 [1-8]. However, the actual number of DCL cases might be higher due to the inconsistent use of chimeric testing on bone marrow transplant patients $[9,10]$. Many factors have been proposed as being associated with the initiation of DCL: donor genetic profile, genetic susceptibility of recipients and/or donors, microenvironment of the host, and therapeutic interventions - such as chemotherapy and some targeted therapies [1, 2, 5, 11, 12]. Although most of the donors are assumed to be genetically normal, there have been cases reported in which the donor had certain constitutional and/or somatic genetic abnormalities overlooked by current pre-transplant screening. In most of these cases, the abnormalities were investigated and detected only after recipients were identified with chromosomal aberration(s) and/or even developed DCL $[1-3,8]$. There also have been case reports of donors remain free of genetic abnormalities, whereas their recipients developed $D C L$ with newly emerging genetic aberrations $[3,8]$.

The molecular genetic events among all reported $D C L$ cases are heterogeneous. Here we report a case of donor cell-derived acute myeloid leukaemia (AML) with 3q26.2 involvement/MECOM rearrangement. We further explore the underlying mechanisms of $D C L$ initiation and progression along with a testing strategy.

\section{Case Report}

The patient was a 65-year-old woman immigrant to the United States who was diagnosed initially with AML in her native country 5 years ago. Her initial bone marrow aspiration and biopsy showed $30 \%$ blasts. Conventional cytogenetic analysis demonstrated a female complex karyotype (no detailed information available) and FISH analysis showed $5 q-, 7 q-,-7$, and +22 as well as loss of one copy of TP53; Sanger sequencing revealed a TP53 G443 frameshift mutation with a variant allele frequency (VAF) of $30 \%$ at that time. The patient was first treated with cytarabine plus daunorubicin and achieved complete remission. A post-chemotherapy bone marrow aspiration and biopsy specimen showed 1\% blast and molecular analysis was negative for TP53 mutation. Conventional cytogenetic analysis and FISH tests were not repeated after therapy. The patient subsequently underwent allogeneic HSCT twice (9 months apart) from the same HLA-matched male sibling. Her first HSCT was complicated with cytomegalovirus (CMV) viremia. It is necessary to point out that according to our clinical notes and the discharge summary from her primary hospital the reason and 
the conditioning details for the second HSCT remain unknown. However, her second HSCT was complicated by graft-versus-host disease (GVHD) mainly in skin and gut. She experienced engraft chimerism and relapse of AML 16 months after the second transplant. She then received azacitidine for 6 courses and achieved a second remission. Seven month later, her disease relapsed again and bone marrow evaluation showed $8 \%$ blasts and an acquired PTPN11 mutation. She was then administered low-dose cytarabine with venetoclax (initially $800 \mathrm{mg}$, then reduced to $600 \mathrm{mg}$ ) for 3 cycles. Due to her persistent disease, she was then referred to our institution.

Initial workup at our institution demonstrated a persistent acute leukaemia with $18 \%$ bone marrow blasts. Flow-cytometry immunophenotyping studies showed aberrant myeloid blasts positive for CD7, CD13, CD33, CD34, CD117, CD123, and HLA-DR. The blasts were negative for CD3 (surface and cytoplasmic) and myeloperoxidase. CD19 was partially positive, but other B cell markers including CD22 and CD79a were negative. The overall immunophenotype supported the diagnosis for AML (Figure 1). Chromosome analysis exhibited a complex karyotype with XY identified in all analysed metaphases: 46,XY, der(2)t(2;3)(p21;q26.2), $\operatorname{der}(3) t(3 ; 3)(q 10 ; q 10) \operatorname{add}(3)(q 26.2) t(2 ; 3)$, $\operatorname{del}(20)(q 11.2 q 13.3)[13]$, indicating that these cells with chromosomal abnormalities were of donor cell origin (Figure 2). Due to unavailability of the donor's genetic fingerprint information, XY FISH tests (probe from Abbott Molecular, Inc., Abbott Park, IL) were performed in both bone marrow and peripheral blood specimens and demonstrated that all cells were $X Y$ (donor) type, suggesting that our patient might have $100 \%$ myeloid engraft. MECOM rearrangement derived from both $\mathrm{t}(2 ; 3)(\mathrm{p} 21 ; \mathrm{q} 26.2)$ and $\operatorname{add}(3)(\mathrm{q} 26.2)$ was detected by interphase FISH, metaphase FISH using MECOM dual-colour, breakapart probe (Leica/Kreatech, Inc., Buffalo Grove, IL) as well as whole chromosomal painting (wcp) for chromosome 3 (Cytocell, Ltd, Tarrytown, NY). For detailed FISH probe and method information, please refer to our previous report [14] (Figure 3). The 20q deletion (referred as del20q thereafter) was also further confirmed by FISH using LSI D20S108 probe (Abbott Molecular, Inc., Abbott Park, IL) [15, 16]. A next generation sequencing (NGS) study of the bone marrow aspirate specimen revealed several mutations with different variant allele frequency (VAF), e.g., BCOR: p.S771* (VAF: 86.8\%); FLT3: p.D835H (VAF: 87.1\%) and PIGA: p.I116V (VAF: <3\%). Our NGS study included an 81-gene panel which were designed specifically for myeloid malignancies. The NGS based sequencing was performed using the Illumina MiSeq (Illumina, San Diego, CA) sequencer as we have reported previously [17]. The FLT3 D835H mutation was further confirmed by a PCR-based method. The patient was then treated with cytarabine, daunorubicin and venetoclax for 1 cycle, and then cytarabine, daunorubicin, venetoclax and midostaturin for another cycle. However, her chromosomal and FISH analyses indicated a persistence of chromosomal abnormalities as well as 3q26.2 involvement/MECOM rearrangement, all in donor cells. NGS after treatment detected several new mutations, e.g., FLT3: p.N676K (VAF: < 3\%); PTPN11: p.A72T (VAF: $29.5 \%$ ) and p.G503A (VAF: < 3\%), in addition to those detected previously. The patient died 6 months later due to severe complications including lactic acidosis, renal failure and fungal pneumonia. 

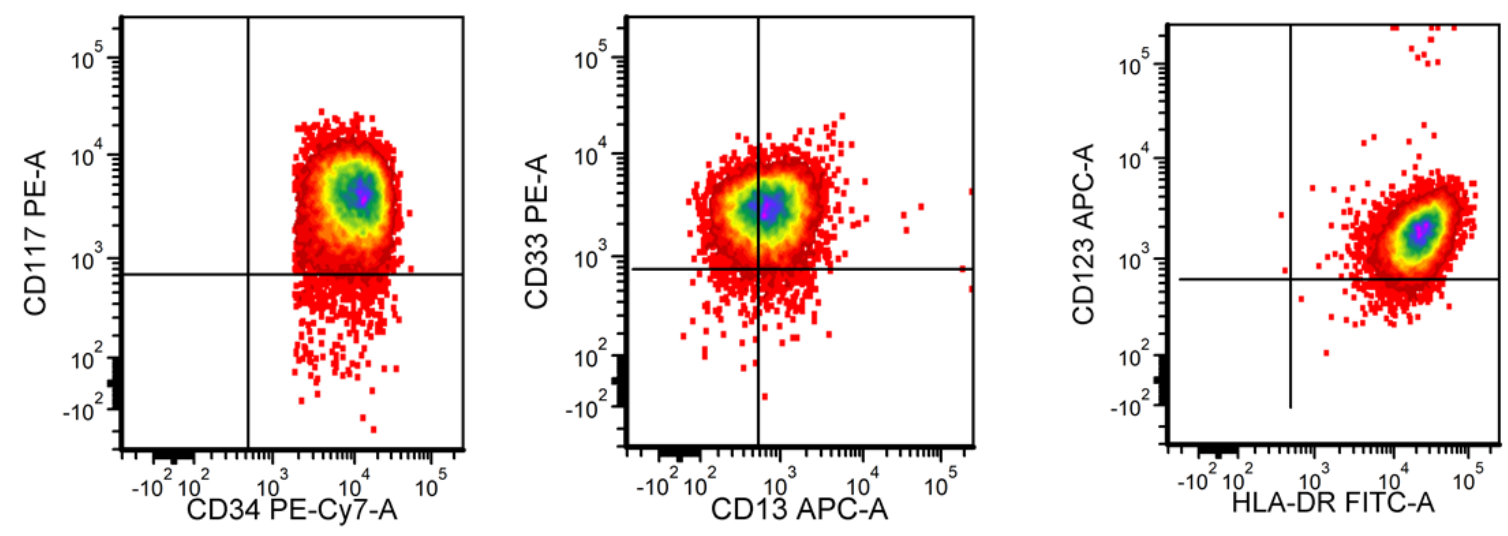

Figure 1 Results of immunophenotype analysis during the initial workup of the patient at our institution. The blasts in the bone marrow aspirate were positive for CD34, CD117, CD13, CD33, CD123 and HLA-DR, diagnostic of AML.
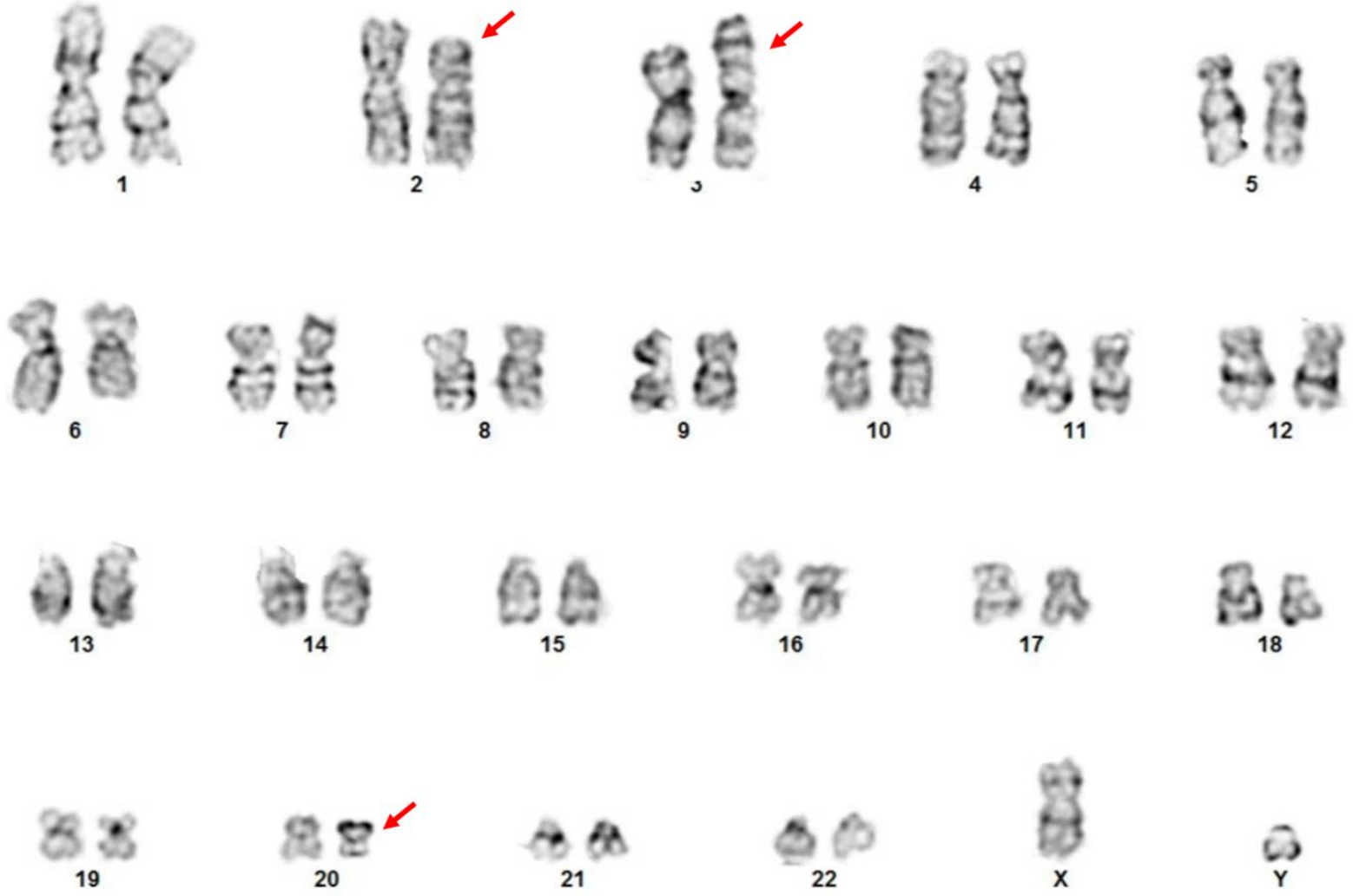

Figure $2 \mathrm{~A}$ representative karyotype image of this cases. The abnormal chromosomes were pointed out with red arrows. The ISCN description of this karyotype result as the following:

$/ / 46, X Y, \operatorname{der}(2) t(2 ; 3)(p 21 ; q 26.2), \operatorname{der}(3) t(3 ; 3)(q 10 ; q 10) \operatorname{add}(3)(q 26.2) t(2 ; 3), \operatorname{del}(20)(q 11.2$ q13.3). 

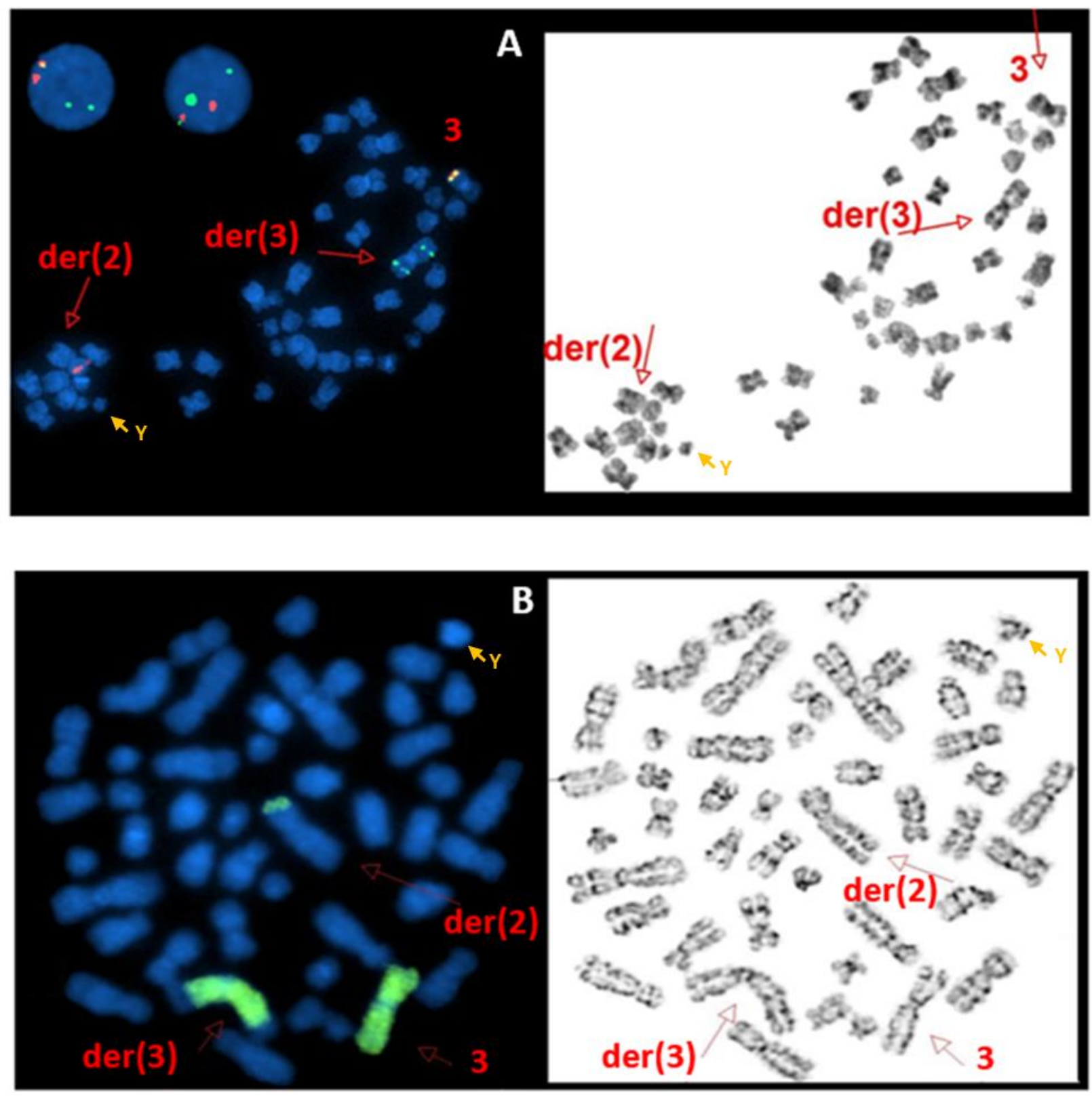

Figure $3 \mathrm{FISH}$ analyses in the initial bone marrow specimen in our institution. A. Interphase and metaphase FISH performed using MECOM (EVI1) Break-apart probe. The two interphases in left upper corner exhibited 1R2G1F signal pattern indicating MECOM rearrangement. Correlating with a metaphase FISH image and G-banding image of the same metaphase (right side), the abnormal chromosome 2, der(2), hosted a 5'MECOM (red) signal; the abnormal chromosome 3, der(3), had two 3'MECOM (green) signals on it, while the normal chromosome 3 had a fusion signal on it. These findings are consistent with the chromosomal analysis results, $\operatorname{der}(2) t(2 ; 3)(p 21 ; q 26.2)$ and $\operatorname{der}(3) t(3 ; 3)(q 10 ; q 10) \operatorname{add}(3)(q 26.2) t(2 ; 3) ;$ B. Whole chromosomal painting (wcp) for chromosomes 3 demonstrated that partial of chromosome 3 materials was located at the ptel of the abnormal der(2), while both ptel and qtel of the abnormal der(3) were not stained. The $Y$ chromosome was pointed out with a golden arrow and a " $Y$ " sign in each image to indicate that the cell was of donor cell origin. 


\section{Discussion}

To the best of our knowledge, this is the first DCL case with confirmed $3 q 26.2$ involvement/MECOM rearrangement reported in the literature [1-4]. Aldoss and Song reported a donor cell AML case with chromosome 3 involvement in 2017 [18]. The karyotype of that case was reported as $46, X X, t(3 ; 13)(p 10 ; q 10)$,inv(11)(p15q23)[13]/46,XX[2] and originated from donor cells, but without 3q26.2 involvement/MECOM rearrangement. The patient achieved complete remission and switched to a normal donor karyotype "46,XX[19]" after chemotherapy. Dias et al [8] reported a case with inv(3)(p26q25) after a sex-matched HSCT. Further intensive investigation revealed that the donor carried this constitutional chromosomal aberration. The recipient in their report remained a complete remission after HSCT and did not develop DCL, but his karyotype switched from 46,XY (recipient type) to 46,XY,inv(3)(p26q25) (donor type) during post-transplant follow-up interval of at least 18 months. Interestingly, the inv(3) was applied as an efficient biomarker for distinguishing donor cells from recipient cells in this circumstance.

Chromosomal aberrations involving 3q26.2 (with or without confirmed MECOM rearrangement) have been reported in cases with various myeloid malignancies such as AML, myelodysplastic syndrome (MDS) and chronic myeloid leukaemia (CML) $[13,14,20]$. These aberrations often present as acquired/additional chromosomal abnormalities, and their emergence usually implies for a poor prognosis. In the patient we report, the 3q26.2 involvement/MECOM rearrangement was detected at the initial diagnosis of donor cell-derived AML. During a follow-up of approximately 6 months, her karyotype analyses and MECOM FISH signal patterns varied slightly (data not included), but the $\mathrm{t}(2 ; 3)(\mathrm{p} 21 ; \mathrm{q} 26.2)$ derived chromosomal aberrations and positive MECOM rearrangement, referred as $\mathrm{t}(2 ; 3) / M E C O M$ rearrangement here, have been consistently detected. In general, the $\mathrm{t}(2 ; 3) / M E C O M$ rearrangement comprised $6-9 \%$ of all MECOM rearrangement-deriving chromosomal aberrations, representing one of the atypical 3q26 aberrations associated with MECOM rearrangements in contrast to the typical inv(3)(q21q26.2)/t(3;3)(q21;q26.2) abnormalities $[13,14,19,20]$. Other studies have suggested that the thyroid adenoma-associated protein gene (THADA) located at 2p21 juxtaposed with MECOM located at 3q26.2 through $t(2 ; 3)(p 21 ; q 26.2)$ translocation and the regulatory elements of THADA can be hijacked by MECOM, resulting in dysregulation of both EVI1 (active component of MECOM) and THADA. The dysregulation is characterized as overexpression of EVII and loss of THADA expression, resulting in a poor prognosis [20-22].

It should be noted that del20q occurred simultaneously with $3 q 26.2$ involvement/MECOM rearrangement after HSCT in this patient. Del20q is a recurrent cytogenetic abnormality observed rarely in the healthy aging population (approximately 0.1\%) [23], and more often in patients with myeloid malignancies including AML (1-2\%), MDS (5-7\%), and in myeloproliferative diseases (MPNs) (up to $10 \%$ ) $[11,15,16]$. Yoon et al [24] reported one case with a donor-derived del20q clone. Although the size of the clone expanded during a follow-up of 18 months in that case, neither disease relapse nor DCL or other donor cell-derived malignancy occurred. Isolated del20q is usually considered as an intermediate prognostic factor in myeloid malignancies [11, 14, 15]. Hence, it seems most likely that 3q26.2 involvement/MECOM rearrangement, rather than del20q, had the most impact on the poor prognosis of donor cell-derived AML in the patient we report.

HSCT is considered as the sole curative option for certain types of AML [25], but post-transplant disease relapse occurs. At the same time, relapsed disease may occasionally differ from the original 
disease at various levels, such as clinical presentation, subtypes of diseases (e.g., myeloid versus lymphoid malignancies due to lineage switch), and/or even different cell of origin (recipient cells versus donor cells). It has been reported that DCL may represent up to $5 \%$ of all post-transplant "relapsed" leukaemia cases, and it is critical to distinguish DCL from the truly relapsed diseases, especially if a second allogeneic HSCT is being considered as the next therapy option. In this case, a post-transplant "relapsed AML" would have been diagnosed if based only on the pathologic and immunophenotypic findings in the bone marrow and peripheral blood. However, the newly identified chromosomal abnormalities including 3q26.2 involvement/MECOM rearrangement were different from the initial disease, at least implying an emergence of different clone(s) of leukemic cells, and the male sex identified by chromosome and XY FISH analyses confirmed origin from donor cells. Mutations of BCOR, FLT3, PIGA and PTPN11 detected after transplant by NGS-based testing were mostly of donor cells, while her previously reported TP53 G443 frameshift mutation was no longer detected.

The factors underlying leukemogenesis of DCL in this case remain unknown. It is believed that multiple factors from recipient, donor as well as therapeutic interventions may play independent and/or synergetic roles in the pathogenesis of donor cell AML (Figure 4) [1-3, 10]. The microenvironment after myeloablative conditioning in the recipient may be carcinogenic to donor cells, and the later can be transformed more easily, especially if any occult pre-leukaemic clones exist, which is normally suppressed by normally functioning immune system of the donor [12]. On the other hand, the recipient's compromised T-cell immunity and microenvironment may have facilitated the growth and expansion of the pre-leukaemic clones to a full-blown AML with distinct cytogenetic and molecular genetic characteristics [4, 7]. Our patient had CMV viremia that occurred after the first HSCT, and GVHD and relapsed AML after the second HSCT from the same donor, indicating a dramatically compromised post-transplant immune system and/or delayed immune reconstitution in the recipient.

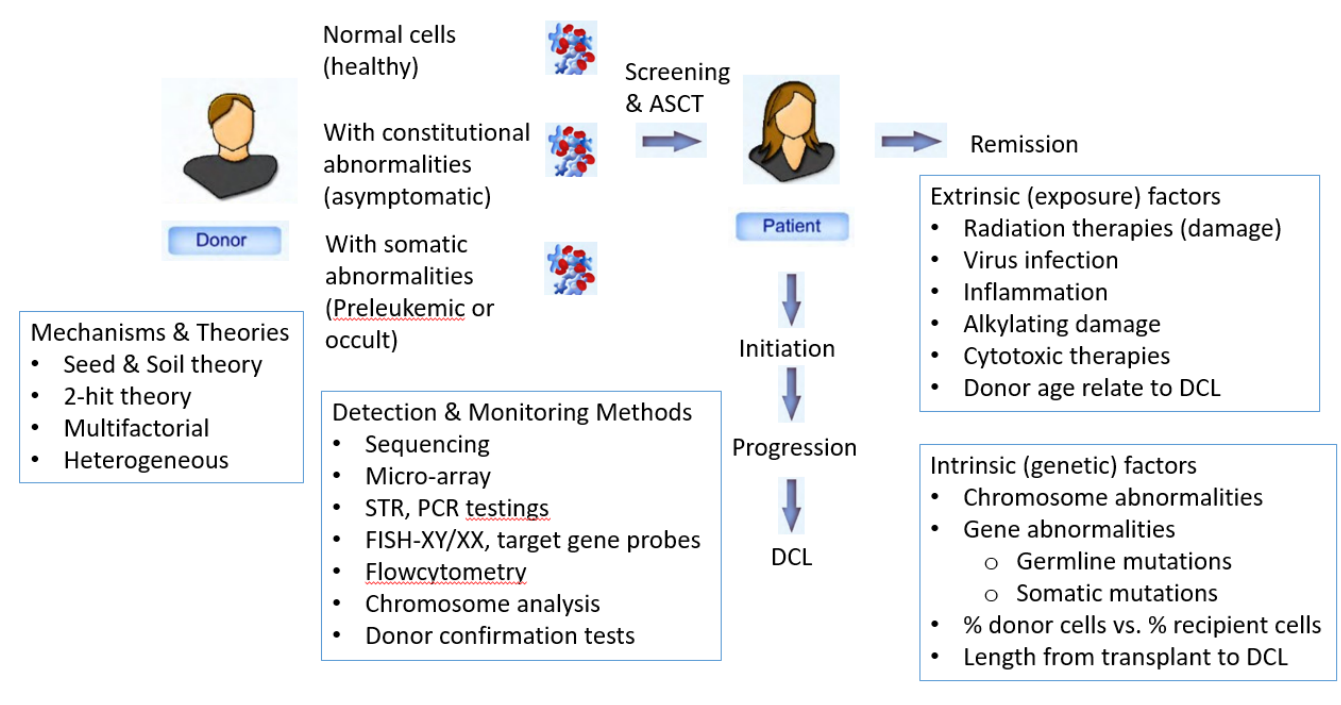

Figure 4. Schematic illustration of hypotheses of leukemogenesis of donor cell leukaemia (DCL) and proposed workup.

According to current clinical practice standards, bone marrow donors are evaluated for general health, but not for any apparent and/or occult comorbid conditions such as constitutional 
chromosomal aberration(s) and/or other cancer-predisposing genetic conditions. Some donors have been reported to carry constitutional chromosomal aberration(s), such as karyotype of 47, XXY or 47,XXY, inversions involving chromosome(s) 3, 9, 10 and 12, trisomy 8, Robertsonian translocation(s), and/or other balanced reciprocal translocation(s) [8, 26]. These chromosomal aberrations are mostly detected after transplant, and the same and/or evolved chromosomal aberrations were detected in the recipients. It remains uncertain whether one or some of these constitutional chromosomal aberration(s) are directly associated with pathogenesis of DCL, but this always triggers the debate about whether bone marrow donors should be more intensively screened, including testing for known tumour-susceptible genetic conditions such as apparent chromosomal aberrations. Clonal haematopoiesis of indeterminate potential (CHIP) is a common occult condition characterized as expansion of age-acquired somatic mutations in hematopoietic lineages that are associated with tumorigenesis, persistence of measurable residual diseases, disease relapse as well as the development of DCL after HSCT in hematologic malignancies. It is believed that CHIP is a novel risk factor for DCL [27], especially if donor and/or recipient are aging individuals. The patient we report was over 60 years old at the time of her second HSCT. TP53 frameshift mutation and PTPN11 mutation have been detected prior to HSCT and during disease relapse post HSCT, respectively. The age of her sibling donor remains unknown to us. Some germline variants, particularly those involving ANKRD26, CEBPA, DDX41, ETV6, GATA2, and RUNX1, have been reported as hereditary predispositions to developing myeloid malignancies [28-30], including donorcell derived malignancies [31]. All these six genes are included in our clinical NGS-based 81-gene leukaemia mutation panel, and none of them had variant alleles.

\section{Conclusions}

We report a case of donor cell-derived AML with 3q26.2 involvement/MECOM rearrangement. This genetic abnormality is often associated with a poor prognosis. Possible factors involved in leukemogenesis of $\mathrm{DCL}$ are also discussed. This case illustrates the importance of considering genetic evaluation of donors as part of screening for HSCT.

\section{Author Contributions}

Conceptualization, Jun Gu, Wei Wang and Zhenya Tang; data curation: Jun Gu, Wei Wang and Zhenya Tang; methodology, Wei Wang, Guilin Tang, Gokce A. Toruner, Ming Zhao, Steven Sfamenos, Zhenya Tang and L. Jeffrey Medeiros; writing-original draft preparation, Jun Gu, Wei Wang and Zhenya Tan; writing-review and editing, Jun Gu, Wei Wang, Ming Zhao, Zhenya Tang, Joseph D. Khoury, and L. Jeffrey Medeiros. All authors have read and agreed to the published version of the manuscript.

\section{Competing Interests}

The authors have declared that no competing interests exist.

\section{References}

1. Ruiz-Argüelles GJ, Ruiz-Argüelles A, Garces-Eisele J. Donor cell leukaemia: A critical review. Leuk Lymphoma. 2007; 48: 25-38. 
2. Wiseman DH. Donor cell leukaemia: A review. Biol Blood Marrow Transplant. 2011; 17: 771789.

3. Dietz AC, DeFor TE, Brunstein CG, Wagner Jr JE. Donor-derived myelodysplastic syndrome and acute leukaemia after allogeneic haematopoietic stem cell transplantation: Incidence, natural history and treatment response. Br J Haematol. 2014; 166: 209-212.

4. Suárez-González J, Martínez-Laperche C, Kwon M, Balsalobre P, Carbonell D, Chicano M, et al. Donor cell-derived hematologic neoplasms after hematopoietic stem cell transplantation: A systematic review. Biol Blood Marrow Transplant. 2018; 24: 1505-1513.

5. Goh KO, Fialkow PJ, Thomas ED, Bryant JI, Neiman PE. Leukaemic transformation of engrafted human cells in vivo. Lancet. 1971; 298: 101-102.

6. Kondo T, Tasaka T, Shimizu R, Hayashi K, Yamada S, Fukuda H, et al. Jumping translocations of $1 q$ in donor cell-derived myelodysplastic syndrome after cord blood transplantation: Case report and review of the literature. Mol Clin Oncol. 2020; 12: 365-373.

7. Bouvier A, Ribourtout B, Francois S, Orvain C, Paz DL, Beucher A, et al. Donor cell-derived acute promyelocytic leukaemia after allogeneic hematopoietic stem cell transplantation. Eur J Haematol. 2018; 101: 570-574.

8. Dias A, Al-Kali A, Van Dyke D, Niederwieser D, Vucinic V, Lemke J, et al. Inversion 3 cytogenetic abnormality in an allogeneic hematopoietic cell transplant recipient representative of a donorderived constitutional abnormality. Biol Blood Marrow Transplant. 2017; 23: 1582-1587.

9. Hertenstein B, Hambach L, Bacigalupo A, Schmitz N, McCann S, Slavin S, et al. Development of leukaemia in donor cells after allogeneic stem cell transplantation--A survey of the European Group for Blood and Marrow Transplantation (EBMT). Haematologica. 2005; 90: 969-975.

10. Dickson MA, Papadopoulos EB, Hedvat CV, Jhanwar SC, Brentjens RJ. Acute myeloid leukaemia arising from a donor derived premalignant hematopoietic clone: A possible mechanism for the origin of leukaemia in donor cells. Leuk Res Rep. 2014; 3: 38-41.

11. Courville EL, Singh C, Yohe S, Linden MA, Naemi K, Berger M, et al. Patients with a history of chemotherapy and isolated del(20q) with minimal myelodysplasia have an indolent course. Am J Clin Pathol. 2016; 145: 459-466.

12. Ogonek J, Juric KM, Ghimire S, Varanasi PR, Holler E, Greinix H, et al. Immune reconstitution after allogeneic hematopoietic stem cell transplantation. Front Immunol. 2016; 7: 507.

13. De Braekeleer M, Le Bris MJ, De Braekeleer E, Basinko A, Morel F, Douet-Guilbert N. 3q26/EVI1 rearrangements in myeloid hemopathies: A cytogenetic review. Future Oncol. 2015; 11: 16751686.

14. Tang Z, Tang G, Hu S, Patel KP, Yin CC, Wang W, et al. Deciphering the complexities of MECOM rearrangement-driven chromosomal aberrations. Cancer Genet. 2019; 233: 21-31.

15. Yin CC, Peng J, Li Y, Shamanna RK, Muzzafar T, DiNardo C, et al. Clinical significance of newly emerged isolated del(20q) in patients following cytotoxic therapies. Mod Pathol. 2015; 28: 1014-1022.

16. Tang G, Lopez JE, Wang SA, Hu S, Ma J, Pierce S, et al. Characteristics and clinical significance of cytogenetic abnormalities in polycythemia vera. Haematologica. 2017; 102: 1511-1518.

17. Tang Z, Toruner GA, Tang G, Yin CC, Wang W, Hu S, et al. Chronic myeloid leukaemia with insertion-derived BCR-ABL1 fusion: Redefining complex chromosomal abnormalities by correlation of FISH and karyotype predicts prognosis. Mod Pathol. 2020; 33: 2035-2045. 
18. Aldoss I, Song JY. Donor-derived myeloid neoplasm post allogeneic hematopoietic cell transplantation. Blood. 2017; 129: 1231.

19. Dowiak AV, Tirado CA. Cytogenetic characterization of myeloid neoplasms with $t(2 ; 3)(p 13-$ 25;q25-29): An analysis of 60 cases. J Assoc Genet Technol. 2017; 43: 64-69.

20. Ottema S, Mulet-Lazaro R, Beverloo HB, Erpelinck C, Herk SV, Helm RV, et al. Atypical $3 q 26 / M E C O M$ rearrangements genocopy inv(3)/t(3;3) in acute myeloid leukaemia. Blood. 2020; 136: 224-234.

21. De Braekeleer M, Guéganic N, Tous C, Le Bris MJ, Basinko A, Morel F, et al. Breakpoint heterogeneity in $(2 ; 3)(\mathrm{p} 15-23 ; \mathrm{q} 26)$ translocations involving EVI1 in myeloid hemopathies. Blood Cells Mol Dis. 2015; 54: 160-163.

22. Trubia M, Albano F, Cavazzini F, Cambrin GR, Quarta G, Fabbiano F, et al. Characterization of a recurrent translocation $\mathrm{t}(2 ; 3)(\mathrm{p} 15-22 ; \mathrm{q} 26)$ occurring in acute myeloid leukaemia. Leukaemia. 2006; 20: 48-54.

23. Ravindran A, He R, Ketterling RP, Jawad MD, Chen D, Oliveira JL, et al. The significance of genetic mutations and their prognostic impact on patients with incidental finding of isolated del(20q) in bone marrow without morphologic evidence of a myeloid neoplasm. Blood Cancer J. 2020; 10: 1-9.

24. Yoon J, Yun JW, Jung CW, Kim HJ, Kim SH. Clonal dominance of a donor-derived del(20q) clone after allogeneic hematopoietic stem cell transplantation in an acute myeloid leukaemia patient with del(20q). J Clin Lab Anal. 2019; 33: e22951.

25. Dholaria B, Savani BN, Hamilton BK, Oran B, Liu HD, Tallman MS, et al. Hematopoietic cell transplantation in the treatment of newly diagnosed adult acute myeloid leukaemia: An evidence-based review from the american society of transplantation and cellular therapy. Biol Blood Marrow Transplant. 2020.

26. Nishihori T, El-Asmar J, Shah B, Hussaini M, Komrokji R, List A, et al. Donor-derived constitutional chromosomal abnormalities after allogeneic hematopoietic cell transplantation: A single-center experience and a review of the literature. Bone Marrow Transplant. 2015; 50: 1388-1392.

27. Burns SS, Kapur R. Clonal hematopoiesis of indeterminate potential as a novel risk factor for donor-derived leukaemia. Stem Cell Rep. 2020; 15: 279-291.

28. Berger G, van den Berg E, Sikkema-Raddatz B, Abbott KM, Sinke RJ, Bungener LB, et al. Reemergence of acute myeloid leukaemia in donor cells following allogeneic transplantation in a family with a germline DDX41 mutation. Leukaemia. 2017; 31: 520-522.

29. Kobayashi S, Kobayashi A, Osawa Y, Nagao S, Takano K, Okada Y, et al. Donor cell leukaemia arising from preleukemic clones with a novel germline DDX41 mutation after allogenic hematopoietic stem cell transplantation. Leukaemia. 2017; 31: 1020-1022.

30. Rafei H, DiNardo CD. Hereditary myeloid malignancies. Best Pract Res Clin Haematol. 2019; 32: 163-176.

31. Mangaonkar AA, Patnaik MM. Hereditary predisposition to hematopoietic neoplasms: When bloodline matters for blood cancers. Mayo Clinic Proc. 2020; 95: 1482-1498. 


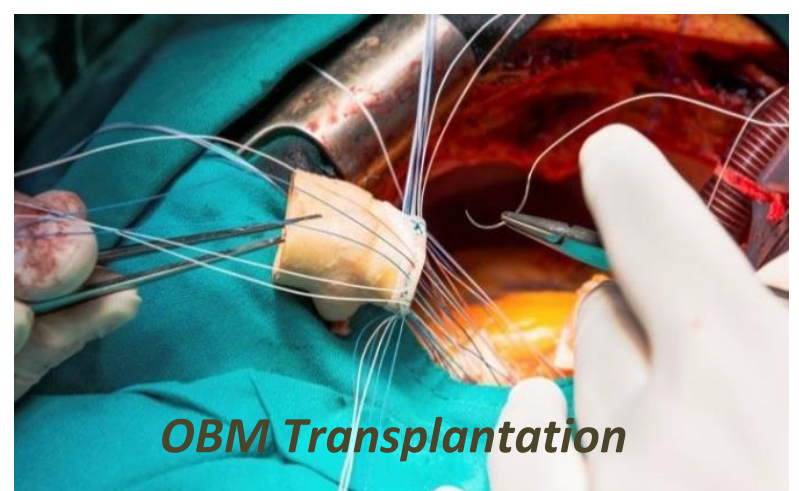

Enjoy OBM Transplantation by:

1. Submitting a manuscript

2. Joining in volunteer reviewer bank

3. Joining Editorial Board

4. Guest editing a special issue

For more details, please visit:

http://www.lidsen.com/journals/transplantation 